\title{
Evaluation of thrombin generating capacity in plasma from patients with haemophilia $A$ and $B$
}

\author{
Yesim Dargaud', Suzette Béguin², Anne Lienhart', Raed Al Dieri², Christine Trzeciak', Jean Claude Bordet', \\ H. Coenraad Hemker², Claude Negrier' \\ 'Laboratoire d'Hémostase Hopital Edouard Herriot, Lyon, France \\ ${ }^{2}$ Synapse Bv, Cardiovascular Research Institute Maastricht, University of Maastricht, The Netherlands
}

\begin{abstract}
Summary
In haemophilia patients, a relationship is usually observed between the clinical expression of the disease and plasmatic factor $\mathrm{VIII/factor} \mathrm{IX} \mathrm{(FVIII/FIX)} \mathrm{activity.} \mathrm{However,} \mathrm{it} \mathrm{is} \mathrm{known} \mathrm{from} \mathrm{clini-}$ cal experience, that some haemophilia patients, despite similar FVIII/FIX plasma levels, could exhibit different bleeding phenotype. After determining preanalytical test conditions, we evaluated the thrombin generation capacity from haemophilia plasma samples in various conditions and the potential usefulness of thrombin generation test (TGT) in haemophilia patients. In a series of 46 haemophilia patients ( 34 haemophilia $A$ and I 2 haemophilia $B$ patients), we found a significant correlation between plasmatic FVIII/FIX levels and endogenous thrombin potential
\end{abstract}

(ETP), peak and time to peak obtained by thrombin generation measurement. In addition, a correlation was found between severe clinical bleeding phenotype and ETP. Our results suggest that TGT could be a promising tool to evaluate haemostasis capacity in patients with haemophilia. Our ex vivo results, obtained 24 hours after FVIII concentrate administration, showed that in patients presenting similar plasmatic FVIIII levels, thrombin generation capacity may be significantly different. These results suggest that in patients with haemophilia, TGT could be useful for individually tailoring prophylactic regimens as well as for adapting clotting factors infusions in surgical situations, in addition to FVIII/FIX plasma clotting activities.

\section{Keywords}

Factor VIII, factor IX, haemophilia, thrombin generation

Thromb Haemost 2005; 93: 475-80

\section{Introduction}

Congenital haemophilia is an X-linked recessive disorder due to mutations in the factor VIII (FVIII) or factor IX (FIX) genes, defined as haemophilia A and haemophilia B. These mutations result in absent or decreased FVIII/FIX procoagulant functions and markedly delayed thrombin generation, despite significant vascular injury (1). In haemophilia patients, a correlation is usually observed between the clinical expression of the disease and plasmatic FVIII/FIX activity. It has however been recognized that some haemophiliacs could experience different bleeding phenotypes despite similar plasma level of FVIII/FIX. The measurement of FVIII/FIX clotting activity has not an excellent predictive value of the individual bleeding risk in haemophilia patients. Recently, Al Dieri et al. (2) showed a correlation between the parameters of the thrombin generation test (TGT) and the clinical bleeding expression in patients with congenital deficiency of factor II, factor V, factor VII, factor X and factor XI. Their results demonstrated that the risk of severe bleeding due to various abnormalities of plasmatic coagulation proteins was invariably accompanied by an aberrant thrombogram. Chantarangkul et al. (3), in a limited series of haemophilia A patients, described a significant correlation between endogenous thrombin potential and $\log$ transformed FVIII plasma level $(\mathrm{r}=0.54$, $\mathrm{p}<0.001)$. This correlation was confirmed in platelet rich plasma from haemophilia patients by Siegemund et al. (4), and recently Varadi et al (5) showed that TGT could be used to monitor the pharmacokinetic properties of by-passing agents in haemophiliacs with inhibitor.

Although haemophilia is theoretically and practically recognised as a disease of thrombin generation, the minimum rate of thrombin generation required to achieve effective haemostasis in haemophiliacs is unknown. Classical clotting tests such as activated partial thromboplastin time (aPTT) and prothrombin time do not reflect thrombin generation entirely, conversely to the classical thrombin generation test. Four major parameters can be assessed in this test: the lag time, the time to peak, the peak height and the endogenous thrombin potential (ETP) (6). The de- 
velopment of an automated form of TGT makes it usable in clinical laboratories (7). We therefore investigated preanalytical conditions required for the application of this test in haemophilia patients, and we evaluated the potential place of TGT in the diagnosis and biological follow-up of haemophilia A and B patients.

\section{Patients, materials and methods}

\section{Patients and volunteers}

Forty six adult haemophilia A and B patients between 20-60 years of age (mean age $=41$ years), routinely treated in the Lyon Comprehensive Haemophilia Care Centre were included, after obtaining informed consent. Among 34 haemophilia A patients, 19 were severe haemophiliacs $(\mathrm{FVIII}<1 \%), 8$ moderate (FVIII $=1-5 \%$ ) and 7 mild (FVIII $>5 \%$ ). Twelve haemophilia B patients were recruited, of whom 4 were severe, 2 moderate and 6 mild. Patients did not indicate any FVIII/FIX concentrate infusion in the week preceding the tests. To establish the severity of haemorrhagic symptoms, the clinical history of each patient was re-examined. Independently from their plasmatic FVIII:C/FIX:C levels, severe bleeders were defined as those presenting the combination of at least 2 of the following indicators: severe radiological haemophilic arthropathy (at least one joint with radiological Pettersson score at 3-4), patients under prophylaxis after recurrent serious bleeding episodes ( $>2$ times per week for at least 3 months) and/or haemophiliacs with history of spontaneous haemorrhages ( $>30$ episodes). The control population comprised 40 apparently healthy males, between 21 and 55 years of age, not using drugs known to affect the coagulation system and recruited from hospital staff.

The TGT parameters from five haemophilia A patients, after administration of $50 \mathrm{IU} / \mathrm{kg}$ FVIII, were compared to classical FVIII pharmacokinetic determination using a one-stage clotting assay.

\section{Blood and platelet poor plasma}

Peripheral venous blood was collected into Vacutainer ${ }^{\mathrm{B}}$ tubes (Becton Dickinson, Meylan, France) containing $0.129 \mathrm{~mol} / \mathrm{L}$ trisodium citrate (1 volume trisodium citrate to 9 volumes blood). Antecubital venipuncture was realised using $21 \mathrm{G}$ needles. For determining the impact of preanalytical conditions, we also tested Vacutainer tubes containing $0.105 \mathrm{~mol} / \mathrm{L}$ trisodium citrate $(\mathrm{n}=3)$ and Monovette (Sarstedt, Orsay, France) containing $0.105 \mathrm{~mol} / \mathrm{L}$ trisodium citrate. The influence of needle calibre $(18 \mathrm{G}$ or $21 \mathrm{G})$ was also tested. Following a double centrifugation at $2500 \mathrm{xg}$ for 15 minutes, platelet poor plasma (PPP) was collected from the upper $1 / 2$ volume of plasma supernatant, quickfrozen and stored at $-80^{\circ} \mathrm{C}$. The absence of platelet and leukocyte in PPP samples was checked with an Advia 120 counter (Bayer Diagnostics, USA). In order to minimize contact activation, polypropylene tubes and pipette tips were used throughout. PPP was always prepared within $30 \mathrm{~min}$ after venipuncture. The prothrombin, the antithrombin and the fibrinogen levels were checked to be in the normal range. For the normal pool, PPP from 5 healthy donors prepared as described above was pooled and stored at $-80^{\circ} \mathrm{C}$.

\section{Clotting factor determinations}

Plasma FVIII and FIX clotting activity was measured by a onestage clotting assay using STA Deficient FVIII and STA Deficient FIX kits (Stago, Asnières sur Seine, France) on STA Compact instrument (Diagnostica Stago, Asnières sur Seine, France) using a standard human plasma calibrated against WHO standard (Dade Behring, Marburg, Germany). The normal range of the FVIII/FIX activities was 50-150 IU/dL. Prothrombin was measured using a one-stage assay with a deficient plasma kit, STA Deficient II (Normal range $=50-150 \mathrm{IU} / \mathrm{dL}$ ). The antithrombin activities were determined using commercial Biophen Antithrombin 5 kit (Hyphen Biomed, Andrésy, France) (Normal range $=80-120 \mathrm{IU} / \mathrm{dL}$ ). Plasma fibrinogen concentration was measured using Fibriquick kit (Biomérieux, Marcy l'Etoile, France), based on the Clauss method (Normal range $=1.8-4 \mathrm{~g} / \mathrm{L}$ ).

\section{Reagents for the thrombin generation test}

Recombinant relipidated tissue factor (TF) Innovin was obtained from Dade Behring (Marburg, Germany). Phospholipid vesicles were obtained from Avanti Polar Lipids (Alabama, USA) and prepared as previously described $(8,9)$. They consisted of 20 mol\% phosphatidylserine, $20 \mathrm{~mol} \%$ phosphatidylethanolamine and $60 \mathrm{~mol} \%$ phosphatidylcholine (7). Hepes-buffered saline contained 20mM Hepes (Sigma Aldrich, l'Ile d'Abeau Chesnes, France), $140 \mathrm{mM} \mathrm{NaCl}$ and $5 \mathrm{mg} / \mathrm{mL}$ bovine serum albumin (BSA) (Euromedex, Souffelweyersheim, France), pH 7.35. A fresh mixture of fluorogenic substrate and $\mathrm{CaCl}_{2}$ was prepared before each experiment. Fluorogenic substrate, Z-Gly-Gly-ArgAMC, was obtained from Bachem (Bubendorf, Switzerland). The mixture of fluorogenic substrate $2.5 \mathrm{mM}$ and $\mathrm{CaCl}_{2} 0.1 \mathrm{M}$ was prepared using buffer containing Hepes $20 \mathrm{mM}$ and $60 \mathrm{mg} /$ $\mathrm{mL}$ BSA, $\mathrm{pH} 7.35$. The Calibrator with the activity of $600 \mathrm{nM}$ human thrombin was obtained from Synapse bv (Maastricht, The Netherlands).

\section{Automated measurement of thrombin generation}

Thrombin generation was measured according to the method described by Hemker et al (7), in a Fluoroscan Ascent fluorometer (Thermolabsystems OY, Helsinki, Finland) equipped with a dispenser. Fluorescence intensity was detected at wavelengths of $390 \mathrm{~nm}$ (excitation filter) and 460nm (emission filter). Briefly, $80 \mu \mathrm{L}$ of PPP were dispensed into the wells of round-bottom 96 well-microtiter plates (Greiner ref.no.65204, Poitier, France). $20 \mu \mathrm{L}$ of a mixture containing TF and phospholipids was added to the plasma sample to obtain a final concentration of $1 \mathrm{pM}$ and $4 \mu \mathrm{M}$ respectively. The starting reagent $(20 \mu \mathrm{L}$ per well $)$ contained fluorogenic substrate and $\mathrm{CaCl}_{2}$. A dedicated software program, Thrombinoscope (Synapse Bv, Maastricht, The Netherlands) enables the calculation thrombin activity and displays thrombin activity with the time. Usually, the measurement lasted 50 minutes. Two wells were needed for each experiment, one well with thrombin generation in the sample and another one with the calibrator (7). All experiments were carried out in duplicate.

\section{Statistical analysis}

Spearman test was applied for correlation analyses. The relation between plasma FVIII/FIX clotting activity and clinical bleeding phenotype of haemophilia patients was evaluated using 
Fisher test. A $p$ value of $<0.05$ was considered statistically significant.

\section{Results}

\section{Test conditions}

The TGT parameters are closely dependent on the concentration of TF. Twenty haemophilic samples were tested with three concentrations of TF $(5,2.5$ and $1 \mathrm{pM})$ and one final phospholipid concentration $(4 \mu \mathrm{M})$. We chose a final concentration of $1 \mathrm{pM}$ for two reasons. Firstly, at this TF concentration, thrombin generation is mainly dependent upon FVIII and FIX clotting factors. Secondly, thrombin generation curves remained flat at this TF concentration in severe haemophiliacs, though traces of thrombin generation occurred after a prolonged lag phase. The most critical step among the preanalytical conditions is certainly the preparation of PPP. Usually, in hospital clinical laboratories, a maximum volume of plasma is harvested in order to limit the number of samples taken from patients. Our results showed that traces of platelets and/or white cells present in the PPP could completely change the TGT results in haemophiliacs and led to over-estimate the thrombin generation capacity (data not shown). If PPP is obtained from the upper $2 / 3$ of the plasma layer, one can omit the additional centrifugation at higher speed (23000xg during 1 hour at $4^{\circ} \mathrm{C}$ ) that is often suggested by research laboratories (2). The quality of PPP obtained can be checked by measuring thrombin generation in the absence of any $\mathrm{TF}$ and phospholipids. Under these conditions no thrombin generation should be obtained.

The sampling material was also evaluated. When PPP was prepared as described above, we did not find significant differences between results obtained with Vacutainer tubes containing 105 or $129 \mathrm{mM}$ trisodium citrate. There was also no significant difference between Vacutainer and Monovette tubes containing each $105 \mathrm{mM}$ trisodium citrate. However, with $1 \mathrm{pM}$ of TF, thrombin generation is also dependent on FXI and contact activation. In order to reduce contact activation, we used hydrophobic polypropylene tubes (less prone to induce activation than polystyrene). $21 \mathrm{G}$ needles were compared to larger $18 \mathrm{G}$ needles, and no difference was noted.

\section{Thrombin generation in inherited haemophilia A and B plasmas}

Thirty four haemophilia A and twelve haemophilia B patients were studied with FVIII/FIX levels ranging between $<1 \mathrm{IU} / \mathrm{dL}$ and $37 \mathrm{IU} / \mathrm{dL}$. The mean values of TGT parameters in severe, moderate and mild haemophiliacs are summarized in Table 1. The thrombogram peak, ETP and time to peak were clearly abnormal in haemophilia patients (Fig. 1).

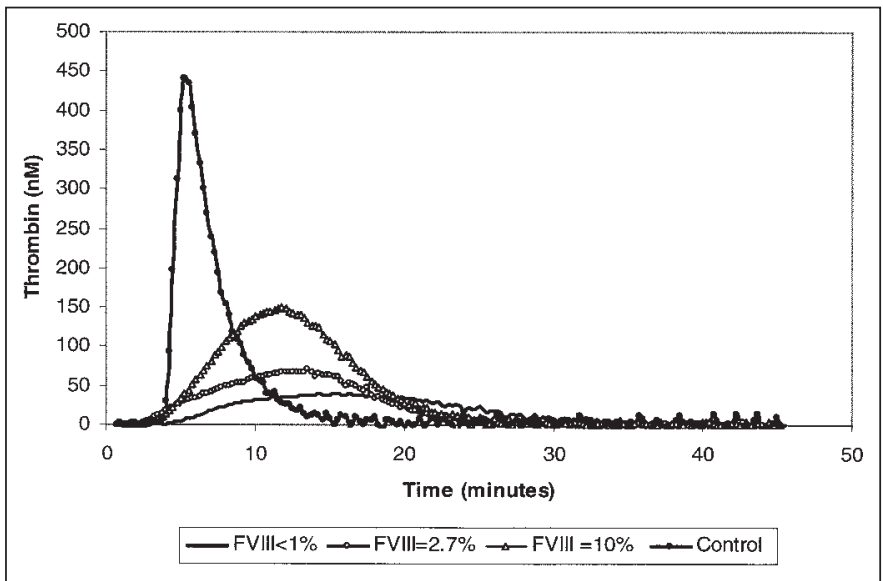

Figure I: Thrombograms obtained with a final concentration of I pM of TF and $4 \mu \mathrm{M}$ of phospholipids in haemophilia $A$ patients. A). severe haemophilia A (FVIII<I\%); B) moderate haemophilia A (FVIII=I-5\%); mild haemophilia A (FVIII >5\%); D). normal control

In haemophilia A patients $(\mathrm{n}=34)$, using Spearman correlation test, we found a significant correlation between plasmatic FVIII levels and ETP $(r=0.53 ; \mathrm{p}<0.0024)$, peak $(\mathrm{r}=0.6$; $\mathrm{p}<0.0006)$ and time to peak $(\mathrm{r}=-0.52 ; \mathrm{p}=0.0026)$ obtained by thrombin generation measurement (Fig. 2). In patients with haemophilia $\mathrm{B}(\mathrm{n}=12)$, a significant correlation was shown between FIX: $\mathrm{C}$ and ETP ( $\mathrm{r}=0.62 ; \mathrm{p}=0.04)$, and peak height $(\mathrm{r}=0.75$; $\mathrm{p}=0.01$ ). In this relatively small haemophilia B series, no statistically significant relationship could be established between FIX: $\mathrm{C}$ and time to peak. Despite these correlations between plasma FVIII/FIX clotting activity and TGT parameters, a relatively large spreading around a general trend could be observed (Fig. 2).

We considered as a severe clinical bleeding phenotype the combination of at least two of the following indicators: severe radiological haemophilic arthropathy (at least one joint with radiological Pettersson score at 3-4), patients under prophylaxis after recurrent serious bleeding episodes $(>2$ times per week for at least 3 months), haemophiliacs with history of spontaneous haemorrhages ( $>30$ episodes). Independently of the FVIII/FIX plasma level, we found that all haemophilia A patients presenting a severe clinical bleeding tendency had an ETP value lower than $50 \%$ of normal (Table 2$)(p<0.0001)$ excepting one haemophilia A patient (patient no. 21) who presented a severe bleeding phenotype but an ETP at 59\% of normal. However, this patient also exhibited a moderate antithrombin deficiency at $69 \%$ (normal values $=80-120 \%$ ). This half-normal ETP was systematically observed at FVIII/FIX concentrations greater than $10 \%$. Conversely, we found an ETP higher than 50\% of normal in two se-
Table I: Quantitative parameters of TGT (ETP, peak height, time to peak) in haemophilia patients and normal control plasmas.

\begin{tabular}{|c|c|c|c|c|c|}
\hline & $\mathrm{n}$ & $\begin{array}{l}\text { ETP } \\
\text { (mean } \pm S D)\end{array}$ & $\begin{array}{l}\text { Peak } \\
\text { (mean } \pm S D)\end{array}$ & $\begin{array}{l}\text { Lag Time } \\
\text { (mean } \pm S D)\end{array}$ & $\begin{array}{l}\text { Time to peak } \\
\text { (mean } \pm \text { SD) }\end{array}$ \\
\hline Severe Haemophilia & 23 & $394 \pm 186$ & $23 \pm 12$ & $6 \pm 2$ & $19 \pm 5$ \\
\hline Moderate Haemophilia & 10 & $657 \pm 205$ & $50 \pm 22$ & $6 \pm 2$ & $15 \pm 3$ \\
\hline Mild Haemophilia & 13 & $1083 \pm 388$ & $125 \pm 67$ & $6 \pm 2$ & $13 \pm 3$ \\
\hline Normal Control Group & 40 & $1495 \pm 175$ & $287 \pm 61$ & $5 \pm 0.7$ & $8 \pm 0.9$ \\
\hline
\end{tabular}


Table 2: Relationship between the clinical bleeding symptoms and TGT parameters in haemophilia patients.

\begin{tabular}{|c|c|c|c|}
\hline Patient & FVIII:C (\%) & Severe bleeding & ETP (\%of normal) \\
\hline I & $<1$ & Yes & 3 \\
\hline 2 & $<1$ & Yes & 21 \\
\hline 3 & $<1$ & Yes & 12 \\
\hline 4 & $<1$ & Yes & 37 \\
\hline 5 & $<1$ & Yes & 36 \\
\hline 6 & $<1$ & Yes & 17 \\
\hline 7 & $<1$ & Yes & 14 \\
\hline 8 & $<1$ & Yes & 19 \\
\hline 9 & $<1$ & Yes & 34 \\
\hline 10 & $<1$ & Yes & 26 \\
\hline 11 & $<1$ & Yes & 46 \\
\hline 12 & $<1$ & Yes & 40 \\
\hline 13 & $<1$ & Yes & 27 \\
\hline 14 & $<1$ & Yes & 22 \\
\hline 15 & $<1$ & No & 26 \\
\hline 16 & $<1$ & Yes & 20 \\
\hline 17 & $<1$ & No & 88 \\
\hline 18 & $<1$ & Yes & 36 \\
\hline 19 & $<1$ & No & 58 \\
\hline 20 & I & Yes & 36 \\
\hline 21 & 1 & Yes & 59 \\
\hline 22 & 1 & Yes & 24 \\
\hline 23 & 2 & Yes & 40 \\
\hline 24 & 2,3 & Yes & 47 \\
\hline 25 & 2,3 & Yes & 42 \\
\hline 26 & 2,4 & Yes & 35 \\
\hline 27 & 2,6 & Yes & 40 \\
\hline 28 & 9,7 & Yes & 40.5 \\
\hline 29 & 10 & No & 100 \\
\hline 30 & 15 & No & 88 \\
\hline 31 & 15 & No & 93.5 \\
\hline 32 & 16 & No & 78 \\
\hline 33 & 20 & No & 51.5 \\
\hline 34 & 30 & No & 65 \\
\hline Patient & FIX:C (\%) & Severe bleeding & ETP (\%of normal) \\
\hline 35 & $<1$ & Yes & 48.5 \\
\hline 36 & $<1$ & Yes & 27 \\
\hline 37 & $<1$ & Yes & 39 \\
\hline 38 & $<1$ & Yes & 14 \\
\hline 39 & 2 & No & 44 \\
\hline 40 & 4 & Yes & 36 \\
\hline 41 & 6 & No & 22 \\
\hline 42 & 7 & Yes & 39 \\
\hline 43 & 7 & No & 74 \\
\hline 44 & 15 & No & 87 \\
\hline 45 & 35 & No & 88 \\
\hline 46 & 37 & No & 100 \\
\hline
\end{tabular}

Figure 3: Pharmacokinetics of thrombin generation capacity (ETP) of 5 PPP samples from haemophilia A patients before and after administration of $50 \mathrm{IU} / \mathrm{kg}$ of FVIII concentrate. The normal ETP range is $1495 \pm 175 \mathrm{nM}$.min (mean $\pm S D$ ).

vere haemophilia A patients (Patients no. 17 and 19) who paradoxically presented a very mild clinical expression of the disease.

In the haemophilia B group ( $\mathrm{n}=12)$ we also observed that patients with a half-normal ETP did not present a severe bleeding tendency $(\mathrm{n}=4)$. Nevertheless, in our relatively limited series, discrepancies were noted in three cases. Patients no.15, 39 and 41 had ETP values clearly less than $50 \%$ of normal but without severe bleeding phenotype.

\section{Effect of FVIII on the thrombin generation of hae- mophilia A patients (ex vivo)}

Blood samples were drawn for the detection of TGT, FVIII, FII, fibrinogen and antithrombin in 5 haemophilia A patients $(2 \mathrm{se}-$ vere, 2 moderate and 1 mild) at pre-infusion (T0), 30 minutes, $1 \mathrm{~h}, 3 \mathrm{~h}, 6 \mathrm{~h}, 8 \mathrm{~h}$ and $24 \mathrm{~h}$ after administration of $50 \mathrm{IU} / \mathrm{kg}$ of FVIII concentrate. 
We observed in all patients, an important increase in peak height and ETP which became normal 30 minutes after FVIII administration. A shortening of the time to peak was also noted in all patients, but no statistically significant change of the lag phase was observed after FVIII injection. Subsequently, a gradual decrease of peak, ETP and time to peak occurred between 3 and 24 hours, but these parameters did not completely return to baseline 24 hours after drug administration.

Our results also showed that 24 hours after FVIII infusion, patients presenting the same residual FVIII: C concentration could have significantly different thrombin generation capacity. As presented on Figure 3, patients $\mathrm{P} 1$ and $\mathrm{P} 2$ who both had a factor VIII level of $18 \%$, exhibited for P1 a thrombin peak of $145 \mathrm{nM}$ and an ETP of 1287nM.min, whereas P2 had a peak of 74nM and an ETP of 885nM.min.

\section{Discussion}

Our results confirm a clear general correlation between circulating FVIII/FIX levels and thrombogram parameters. In haemophilia, with defective plasma FVIII or FIX concentrations, the peak height and the ETP are decreased, the time to peak is longer, but the lag time does not lengthened. These results are in agreement with previously reported data in individuals with rare inherited coagulation disorders. Al Dieri et al (2) showed that the lag time was not systematically prolonged in these patients. The time to peak is evidently dependent upon the lag time, but also reflects the efficacy and the velocity of the prothrombin conversion. Our results showed that this parameter decreased when FVIII/FIX concentrations increased, demonstrating that in haemophiliacs thrombin generation is delayed compared with healthy subjects.

Factor VIII/Factor IX clotting activity assays have a relatively poor predictive value of the individual bleeding risk in haemophilia patients. It is well known from clinical observations that some haemophilia patients, despite similar FVIII/FIX concentrations and/or with identical mutations, exhibit different clinical expression of the disease (10). An association with thrombophilia risk factors has been evoked in these cases (11). Considering the high prevalence of factor $\mathrm{V}$ Leiden mutation $(2-7 \%)(12,13)$, it is possible that some haemophilia patients also carry this thrombophilic mutation $(14,15)$. Factor V Leiden mutation, as well as the majority of other thrombophilia risk factors, increase thrombin generation (16-18). Thrombin generation assay, which reflects hypo- and hypercoagulability properties, might bring additional information to those provided by genotype analysis and plasma clotting activity. In our series, approximately $25 \%$ of haemophiliacs did not present a close correlation between FVIII/FIX levels and TGT results. In the majority of these patients we noted a discrepancy between FVIII/FIX activity and clinical expression of the disease.

Indeed, two severe haemophilia A patients $(\mathrm{FVIII}<1 \%)$ who had a very attenuated bleeding phenotype (patients no. 17 and 19), had both an ETP higher than 50\% (Table 2). In patient no. 17 who had an ETP at $88 \%$ of normal, the haemophilia diagnosis was done fortuitously at the age of 44 years because of an abnormal aPTT result. This patient had never experienced any severe bleeding. Patient no.18, his brother, had an ETP at $36 \%$ of nor- mal, and he frequently experienced bleeding responsible for an haemophilic arthropathy. In these patients, who had the same missense mutation (Thr 118 Ile in exon 4), TGT mirrored a different clinical expression. In our series, a mild haemophilia A patient (no. 28, FVIII:C=9.7 UI/dL) with a severe haemophilic arthropathy, showed a relatively poor thrombin generation (ETP $=40.5 \%$ of normal), whereas another mild haemophilia A patient with similar plasma FVIII concentration (no. 29, FVIII $=10 \%$ ) but without severe haemorrhages had normal thrombin generating capacity (ETP $=100 \%$ of normal). An antithrombin deficiency associated with severe haemophilia A was fortuitously detected in another patient (patient no. 21), and the combination of both deficiencies likely influenced the TGT results. We systematically checked the normality of prothrombin, antithrombin and fibrinogen levels, which might influence thrombin generation.

We noted that patients with a severe clinical bleeding tendency usually had an ETP $<50 \%$ of normal, independently from FVIII:C/FIX:C plasma concentrations. However, some negative results were also found. In this series, 3 haemophiliacs with a severely decreased ETP presented mild bleeding symptoms. One of them (patient no. 15) was under immune tolerance therapy which could explain the rarity of bleeding episodes. The second (patient no. 39) was a moderate haemophilia B patient (FIX:C $=2 \%$ ) who was treated with carbamazepine for many years. Some cases of venous thromboses have been previously reported with carbamazepine therapy (19-21). Recently, Ulrich et al. (22) showed that in vitro basal platelet calcium concentration, as well as thrombin induced peak of cytosolic calcium concentration, was significantly higher in platelets incubated with carbamazepine as compared with control platelets. Taken together, these data might at least partly explain the discordance between low TGT results in PPP and the clinical expression of the disease. We did not find, for the third patient (patient no. 41), any environmental reason explaining the discrepancy. However, Siegemund et al (4) recently showed an important role of platelets in haemophilia B patients which suggests that our information based on PPP only might be incomplete for this particular haemophilia B patient. Our results suggest that in addition to FVIII/FIX plasma measurements, TGT could be a precious tool for physicians in the management of haemophilia $\mathrm{A}$ and $\mathrm{B}$, but it should be emphasized that the quality of the results are critically dependent upon preanalytical conditions.

Platelet-poor plasma preparation is the most important preanalytical step, and double centrifugation is considered as essential. The transfer of plasma between two centrifugations should be done using hydrophobic material. The final PPP has to be leukocyte and platelet free. Recently, Giansily-Blaizot et al (23) reported important discrepancies in TGT results of congenital FVII deficient patients. In this multi-centre study, the authors evoked that these variations could be probably explained by differences in the preanalytical conditions used in different centres. Our systematic evaluation of preanalytical conditions support their hypothesis, because PPP was obtained from only one centrifugation at 2000xg during $20 \mathrm{~min}$. In their study, the lag-time values were extremely short ( 0.5 minute for the normal control PPP), which might be explained at least in part by the unexpected presence of TF bearing cells. 
Finally, our results show that TGT can be used for monitoring pharmacokinetics of FVIII. We found that an injection of $50 \mathrm{IU} /$ $\mathrm{kg}$ of FVIII was able to restore normal thrombin generation in haemophilia A patients 30 minutes after drug administration, subsequently to the increase in plasma FVIII concentration. Nevertheless, some patients with similar FVIII levels by coagulation assay may have significantly different TGT results (e.g. patients $\mathrm{P} 1$ and $\mathrm{P} 2$ presenting the same residual FVIII:C concentration at 18\%, 24 hours after FVIII infusion). This result suggests that TGT could also be of interest in the follow -up of haemophiliacs under prophylaxis. Protocols for haemophilia A prophylaxis usually comprise $2-3$ administrations per week of weight-adjusted doses. The present regimens will tend to remain on the safe side and may have a tendency of overdosing, since the minimum level of FVIII/FIX that will prevent bleeding physiologically is not known. This issue may have an important impli- cation for factor consumption and treatment cost. Thrombin generation associated with plasma FVIII/FIX measurements might represent a promising tool to evaluate more precisely the efficacy of a prophylactic regimen and thus to improve the overall cost-effectiveness of anti-haemophilic treatments. Further studies in this field remain to be done.

In conclusion, our results suggest that TGT can represent a helpful guide in the evaluation of the coagulation capacity of haemophilia patients and in the optimisation of FVIII/FIX dosage to be administered. In order to approach in vivo conditions more closely, measurements in platelet-rich plasma might be even more informative. The results from a relatively limited group presented here justify a larger multicentre study in order to firmly establish the correlations between clinical expression of the disease, function test (i.e. TGT) and substitution therapy.

\section{References}

1. Mannucci PM, Tuddenham EG. The hemophilias from royal genes to gene therapy. N Engl J Med 2001; 344: 1773-9.

2. Al Dieri R, Peyvandi F, Santagostino E, et al. The thrombogram in rare inherited coagulation disorders: its relation to clinical bleeding. Thromb Haemost 2002; 88: 576-82.

3. Chantarangkul V, Clerici M, Bressi C, et al. Thrombin generation assessed as endogenous thrombin potential in patients with hyper-or hypo-coagulability. Haematologica 2003; 88: 547-54.

4. Siegemund T, Petros S, Siegemund A, et al. Thrombin generation in severe haemophilia $\mathrm{A}$ and $\mathrm{B}$ : the endogenous thrombin potential in platelet-rich plasma. Thromb Haemost 2003; 90: 781-6.

5. Varadi K, Negrier C, Berntorp E, et al. Monitoring the bioavailability of FEIBA with a thrombin generation assay. J Thromb Haemost 2003; 1: 2374-80.

6. Hemker HC, Beguin S. Phenotyping the clotting system. Thomb Haemost 2000; 84: 747-51.

7. Hemker HC, Giesen P, AlDieri R, et al. Calibrated automated thrombin generation measurement in clotting plasma. Pathophysiol Haemost Thromb 2003; 33: 4-15.

8. Hope MJ, Bally MB, Webb G, et al. Production of large unilamellar vesicles by rapid extrusion procedure. Characterization of size distribution, trapped volume and ability to maintain a membrane potential. Biochim Biophys Acta 1985; 812: 55-65.
9. Hedegaard E, Jensen B. Nano-scale densitometric quantitation of phospholipids. J Chromatogr 1981; 225: $450-4$.

10. Ghosh K, Shetty S, Mohanty D. Milder clinical presentation of hemophilia A with severe deficiency of factor VIII as measured by one-stage assay. Haemophilia 2001; 7: 9-12.

11. Nowak-Gottl U, Escuriola C, Kurnik K, et al. Haemophilia and thrombophilia. Hamostaseologie 2003; 23: $36-40$

12. Dahlback B, Carlsson M, Svensson PJ. Familial thrombophilia due to a previously unrecognised mechanism characterized by poor anticoagulant response to activated protein $\mathrm{C}$ : prediction of a cofactor to activated protein C. Proc Natl Acad Sci USA 1993; 90: 1004-8.

13. Bertina RM, Koeleman BP, Koster T, et al. Mutation in blood coagulation factor $\mathrm{V}$ associated with resistance to activated protein C. Nature 1994; 369: 64-7. 14. Nichols WC, Amano K, Cacheris PM et al. Moderation of hemophilia A phenotype by the factor $\mathrm{V}$ R506Q mutation. Blood 1996; 88: 1183-7.

15. Lee DH, Walker IR, Teitel J, et al. Effect of the factor V Leiden mutation on the clinical expression of severe hemophilia A. Thromb Haemost 2000; 83: 387-91. 16. Curvers J, Thomassen MC, Rimmer J, et al. Effects of hereditary and acquired risk factors of venous thrombosis on a thrombin generation-based APC resistance test. Thromb Haemost 2002; 88: 5-11
17. Wielders S, Mukherjee M, Michiels J, et al. The routine determination of the endogenous thrombin potential, first results in different forms of hyper- and hypocoagulability. Thromb Haemost 1997; 77: 629-36. 18. Tans G, van Hylckama Vlieg A, Thomassen MC, et al. Activated protein $\mathrm{C}$ resistance determined with a thrombin generation-based test predicts for venous thrombosis in men and women. Br J Haematol 2003; 122: 465-70.

19. Dacosta A, Mismetti P, Tardy B, et al. Thrombosis and carbamazepine: hazard or causality? Therapie 1994; 49: 355-6.

20. Simon JP, Hanesse B, Trechot P, et al. Repeated thromboses during treatment with carbamazepine. Therapie 1993; 48: 491.

21. Nakaso K, Shimoda M, Yasui K, et al. A case of superior sagittal sinus thrombosis following long-term medication with carbamazepine. Rinsho Shinkeigaku. 2000; 40: 617-20.

22. Ulrich ML, Rotzinger S, Asghar SJ, et al. Effects of dextroamphatamine, lithium chloride, sodium valproate and carbamazepine on intraplatelet $\mathrm{Ca}^{2+}$ levels. $\mathrm{J}$ Psychiatry Neurosci 2003; 28: 115-25.

23. Giansily-Blaizot M, Al Dieri R, Schved JF. Thrombin generation measurement in factor VII-depleted plasmas compared to inherited factor VII-deficient plasmas. Pathophysiol Haemost Thromb 2003; 33: 36-42. 